\title{
Radiation dose reduction in computer assisted navigation for functional endoscopic sinus surgery - cadaver head experiments and clinical implementation*
}

\author{
P. Dubach, A. Eichenberger, M. Caversaccio
}

Department of Otorhinolaryngology, Head and Neck Surgery, Inselspital, Bern University Hospital, and University of Bern, Switzerland

SUMMARY Objectives: Computed tomography based navigation for endoscopic sinus surgery is inflationary used despite of major public concern about iatrogenic radiation induced cancer risk. Studies on dose reduction for CAS-CT are almost inexistent. We validate the use of radiation dose reduced CAS-CT for clinically applied surface registration.

Methods: Dose reduced CAS-CT of mineral salt fixed, human cadaver heads with 9.6, 6, 4, 2 and $1.1 \mathrm{mGy}$ were compared with the reference dose at $65 \mathrm{mGy}$ CTDI (CT-Dose Index). For each CT dose with different surface resolution, the precision of the soft touch registration was measured with target registration error (TRE). In a practical step, dose reduced protocols were tested for 12 months.

Results: Using surface registration at highest and lowest doses, TRE did not differ significantly for registration accuracy. Protocols tested preserved technical registration accuracy and the pragmatics of dose reduction was limited only by different needs for picture quality of the individual surgeon, use for uncomplicated or revision surgery, and reserve for other unexpected factors (movement artifacts).

Conclusions: The accuracy of today's surface registration technology was not the limit for dose reduction. It is the amount of diminished picture quality tolerated by the individual surgeon and the question of how much of the ever refined radiological picture resolution is necessary at all. For the majority of operations, consensus for a significant 6-fold radiation dose reduction from $65 \mathrm{mGy}$ to $9.6 \mathrm{mGy}$ CTDI could be realized illustrating a big potential for similar approaches in other institutions.

Key words: functional endoscopic sinus surgery, computer assisted surgery, image guided surgery, navigation, surface registration, computed tomography, radiation dose reduction, Thiel, mineral salt fixation

\section{INTRODUCTION}

Since the inception of Computed Tomography (CT) in the 1970s, image quality, range of clinical applications and logistical availability increased almost exponentially. According to US American studies, there was an about 600\% increase in the number of examinations in both adults and children in a 10 - year period ${ }^{(1)}$. Radiation dose exposure has the potential of various risky side effects ranging from radiation induced cataract formation to mutagenic or teratogenic damage. The increasing number of examinations and the related exposure to radiation is becoming not only a major concern in the daily press ${ }^{(2,3)}$ but also an issue in epidemiologic studies correlating more widespread use of CT with increased life-time risk of death from radiation induced cancer ${ }^{(4)}$. This is especially important for chil- dren or patients who are at great risk of having repeated follow up CT scans in their lifetime ${ }^{(5)}$.

In ENT, applications of Computer Assisted Navigated Surgery (CAS) are rapidly growing, especially in Functional Endoscopic Sinus Surgery (FESS), skull base surgery and otological surgery for safety, didactic as well as medico legal reasons ${ }^{(6,7)}$. Although FESS with MR based CAS is feasible ${ }^{(8)}$, preoperative acquisition of CT-based datasets for navigation is still standard, implying radiation dose induced hazards to the patient. This study tries to give practical solutions to answer the growing public concern about a contribution of unnecessary radiation dose exposure caused by the ENT specialist.

In rhinology, dose reduced protocols for conventional scans (i.e. sinusitis) do exist ${ }^{(9-12)}$. However, only recently was radiation 
dose reduction assessed for the first time for CAS-CT in FESS with a clinical survey and preclinical experiments. Technical accuracy with paired-point registration for dose reduced CASCT were tested under laboratory conditions with screw fixed registration points. The survey showed an inter-institutional 18fold difference in radiation dose exposure for CAS-CT protocols and pointed theoretically to a significant potential for dose reduction ${ }^{(13)}$.

Despite the promising results of rigid registration, screw fixed fiducials are used in neurosurgery but are hardly tolerable in ordinary FESS ${ }^{(14)}$. Hence, we tested the feasibility of accurate registration and navigation with dose reduced CAS-CT for the clinically used surface registration. In a second step, validated dose reduced protocols preserving registration accuracy were tested by pragmatic step by step dose reduction in real surgery.

\section{MATERIALS AND METHODS}

\section{Scanning protocol}

Registration and navigation was performed by the optically guided Vector Vision System (BrainLAB ${ }^{\circledR}$, Feldkirchen, Germany) routinely used in our operating room.

For CAS-CT, overlapping $1.25 \mathrm{~mm}$ slices with a field of view (FOV) of $20 \mathrm{~cm}$ were acquired with a Lightspeed Ultra Scanner ${ }^{\circledR}$ (General Electric, Milwaukee, WI, USA). Images were reconstructed in edge-enhancing algorithm, optimal for bony contours. The field of view included the facial surface from the upper edge of the frontal sinus to the upper tooth row, including the tip of the nose, allowing reliable preoperative registration. Radiation dose was measured as CT Dose Index (CTDI) in $\mathrm{mGy}$, as this can be estimated by dosimetry for a single CT slice

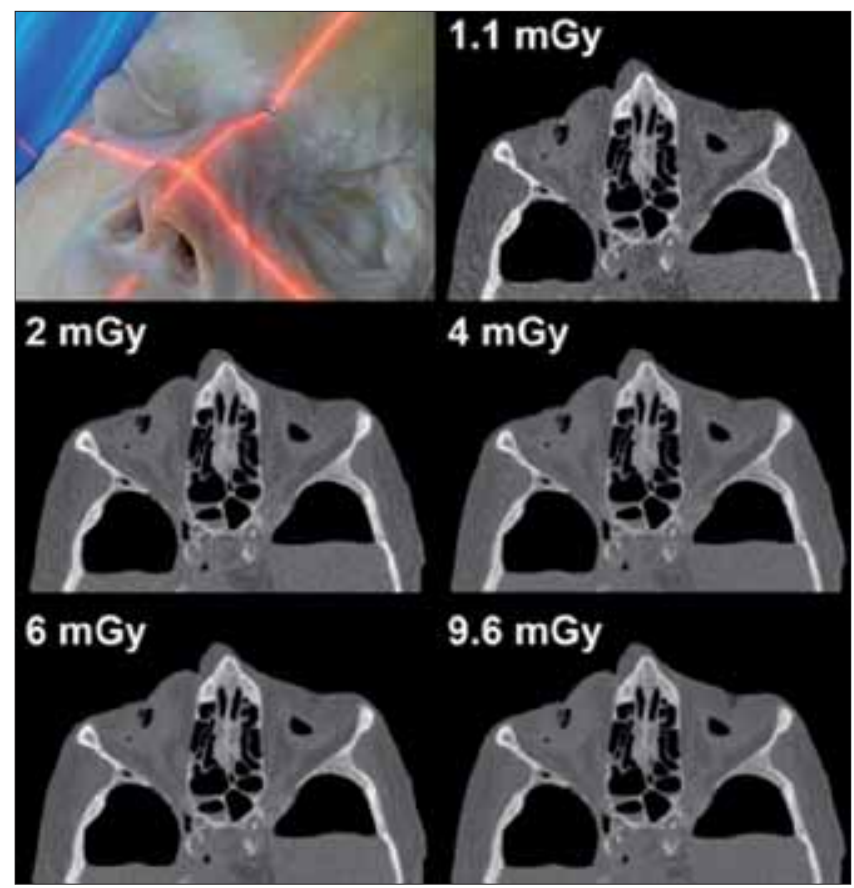

Figure 1. Thiel cadaver head in the scanner and representative axial view of the 5 dose reduced CAS-CT protocols used for surface registration. and approximated with theoretical models. CTDI is a technical parameter provided by all CT manufacturers, but it is not directly related to an individual organ dose. Thus, we added an estimate of the effective dose measured in $\mathrm{mSv}$, as this better reflects a generic estimate of the overall harm to the patient caused by radiation dose exposure.

The influence of dose reduction of CAS-CT on registration accuracy at 9.5, 6, 4, 2 and $1.1 \mathrm{mGy}$ CTDI (Figure 1) was tested. As a reference dose, the initially used high dose protocol (120 $\mathrm{kV}, 210 \mathrm{mAs}$ corresponding to $65 \mathrm{mGy}$ CTDI) was included as well.

For surface registration with soft touch (s-touch) technology, a pointer was used to tip on 30 skin surface points for correlation with the virtual surface in the CAS-CT image.

Accuracy was estimated ${ }^{(15-17)}$ using the Target Registration Error (TRE) of 5 small screws drilled into the bone of the fronto-zygomatic suture (screw 1,2), the nasion (screw 3), the nasal spine (screw 4) and into the left pteryon (screw 5 in Figure 2 left up). The head of the screws had a central pit for the pointer tip used for accuracy measurement (Figure 2, right bottom). The center of the pits was marked with the system software iPlan ${ }^{\circledR}$ pre-procedurally at maximal magnification. Putting the pointer tip exactly at the marked location, TRE is calculated ${ }^{(18)}$. This value indicates the maximal deviation between patient and image data ${ }^{(19)}$.

In a second practical part, radiation dose for CAS-CT was reduced stepwise in clinical routine to the in vitro estimated values tolerable for salvage surgery. Surgeons (4 fellows and the senior author of the paper; median experience in rhinosurgery 3 years (range 1-15) were first offered ample opportunity to test

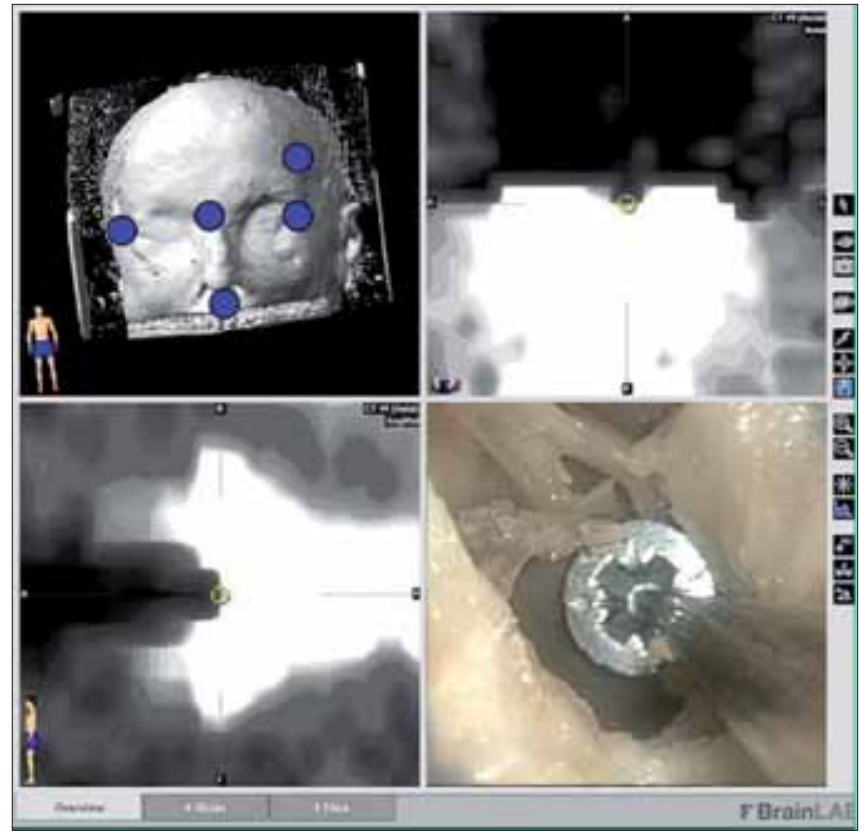

Figure 2. Screw fixed landmarks (5 points seen in the upper left figure) for accuracy measurement in TRE with the pointer at the center of the screw groove (bottom right). Highest magnification of the screw pit in the software iPlan (bottom left and top right). 
Table 1. Surgeon's objections limiting pragmatic dose reduction in clinical practice.

\begin{tabular}{|c|c|c|}
\hline \multicolumn{3}{|c|}{ Arguments limiting pragmatic dose reduction } \\
\hline Aspects & Dangers & Example \\
\hline Diagnostic & $\begin{array}{l}\text { Risk of missing alternative diagnosis, unsuitable for } \\
\text { emergency situations }\end{array}$ & $\begin{array}{l}\text { Extent of tissue infiltration by tumours need } \\
\text { of contrast media application }\end{array}$ \\
\hline $\begin{array}{l}\text { Navigation } \\
\text { (Signal to Noise problem) }\end{array}$ & Problems of identifying thin bony septa of landmarks & Damage to the carotid canal or ethmoidal fovea \\
\hline Registration & $\begin{array}{l}\text { Too narrow field of view with insufficient midface contours } \\
\text { for registration }\end{array}$ & $\begin{array}{l}\text { Inadequate contour of the nose compromises } \\
\text { the area for registration }\end{array}$ \\
\hline Image stability & Reserve for other factors reducing the signal-to-noise ratio & $\begin{array}{l}\text { Movement artifacts of non compliant patient } \\
\text { diminishing picture quality }\end{array}$ \\
\hline Logistic & $\begin{array}{l}\text { Acceptance for a wide group of users (beginners, irregular users, } \\
\text { external consultants) }\end{array}$ & $\begin{array}{l}\text { Satisfying picture quality for change of the } \\
\text { operation to another surgeon }\end{array}$ \\
\hline
\end{tabular}

navigation with dose reduced CAS-CT on the cadaver heads. They defined an individual limit to dose reduction at which they still felt $100 \%$ confident in the navigation quality ${ }^{(13)}$. During a 12 months period, validated CT protocols preserving technical registration accuracy were used for surgery with progressive CASCT dose reduction.

\section{Statistical analysis}

For descriptive statistics of accuracy measurements, the box blot for TRE, depending on CT dose, was constructed. For inferential statistical analysis, a non-parametric Wilcoxon signed rank test with Bonferroni correction for multiple comparisons was used to compare accuracy (TRE) depending on CT radiation dose used.

\section{RESULTS}

\section{Experiments with cadaver heads}

Surface registration with the s-touch method was possible in pilot studies on a dummy as well as on the cadaver heads. Lifelike plasticity of the Thiel fixed skin surface allowed comparable fast and effective s-touch registration to real surgery. Accuracy measurements for all CT radiation doses (Figure 3) showed an overall mean TRE of $2.09 \mathrm{~mm}$ (range of mean values 1.7-2.5 $\mathrm{mm}$ ), which was comparable to benchmark values for surface registration techniques cited in the literature ${ }^{(18,20-27)}$. The accuracy of TRE following registration by CAS-CT with the highest dose (i.e. $65 \mathrm{mGy}$ CTDI) and with lowest dose (1.1 mGy CTDI) was not significantly different $(\mathrm{p}>0.05$, Wilcoxon signed rank test, Bonferroni corrected).

\section{Pragmatic dose reduction in clinical practice}

During annual training courses and in laboratory pilot experiments, the 5 surgeons had ample opportunity to gain experience with dose reduced images for registration and intra-operative navigation in FESS. Using s-touch registration instead of rigid registration clearly facilitated the transfer of the in vitro optimized dose reduction from $65 \mathrm{mGy}(2.2 \mathrm{mSv})$ to $9.5 \mathrm{mGy}(0.3$ $\mathrm{mSv}$ ) in everyday practice. Time consumption for both s-touch registration and actual operation was not bigger for registration with dose reduced CAS-CT compared with high dose CAS-CT. Because of the affiliation to the national competence center for
Computer-Aided and Image-guided Medical Interventions (www.co-me.ch), CAS is regularly performed for FESS at our institution. During the study period of 12 months, radiation dose reduced CAS-CT were regularly performed in standard situations, in revision surgery or if scans had to be repeated preoperatively due to insufficient imaging from other radiology departments. In this period, 104 CAS based FESS operations were performed: 84 with CAS-CT at $9.6 \mathrm{mGy}$ and 20 (11 operations for complications, 9 oncologic procedures) at $65 \mathrm{mGy}$ CTDI. Thus 4 out of 5 patients could take advantage of reduced radiation dose exposure. Consensus of all participating surgeons was not to reduce the dose at all costs. Debate started for dose reduction from 9.6 to $6 \mathrm{mGy}$ CTDI and arguments limiting further reduction are depicted in Table 1.

\section{DISCUSSION}

The use of CAS technology is growing exponentially for the sake of safety, for protection against legal concerns, as surgical training tool ${ }^{(6)}$ or for new domains as for guidance and control of brain mapping strategies ${ }^{(28,29)}$. Apart from conventional CT scanners, volume tomography offers advances in technology in dose reduction. On the other hand, it remains to be seen whether the increase in availability and new fields of applications, i.e. additional intra-operative imaging ${ }^{(30)}$, do not outweigh

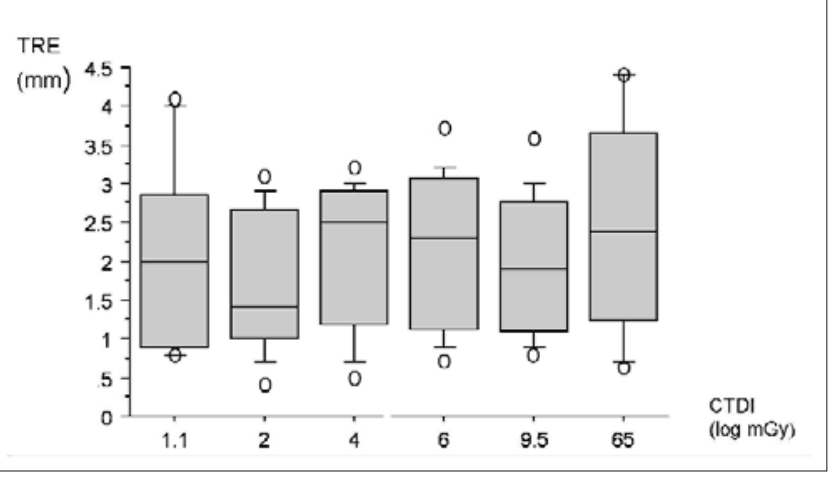

Figure 3. Box plot of accuracy measurements (TRE) of screw fixed landmarks in mm using s-touch registration. Each row shows the TRE of the landmarks for each CAS-CT dose. Lines dividing the rectangle depict the mean value of TRE, box margins indicate 25 percentile and 75 percentile values. Open circles indicating the range of the values. 


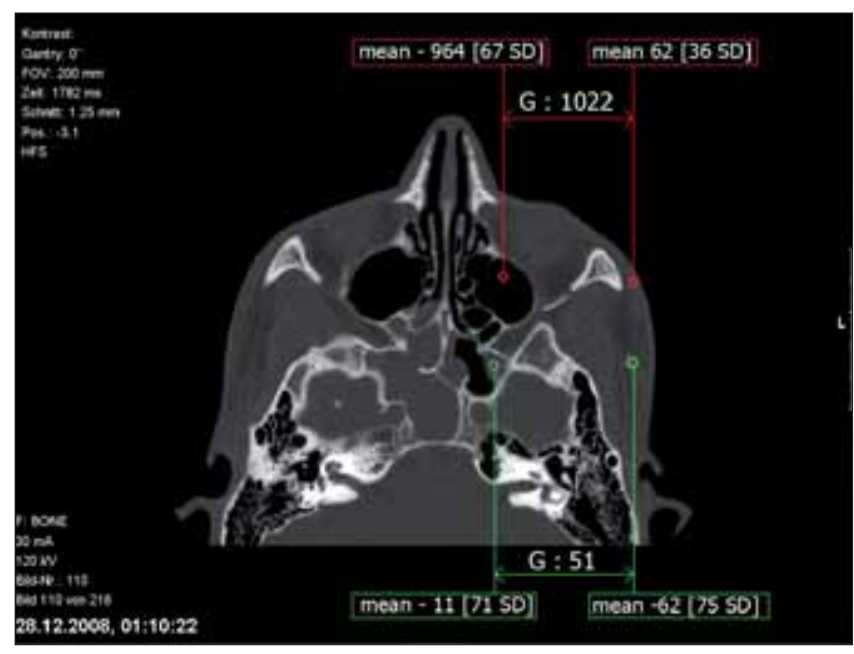

Figure 4. CT radiation dose absorption of different tissue components according to the Hounsfield scale. Favorable for dose reduction using surface registration methods is the high gradient $(\mathrm{G})$ between air and soft tissue (G:1002, data at the top of the image) as well as well as the high gradient between bone and soft tissue for the bone window based navigation in FESS. On the other hand, gradients on the Hounsfield scale between soft tissue and fluid (G:51, data at the bottom of the image) are smaller, rendering soft tissue based surgery more vulnerable to dose reduced $\mathrm{CT}$ imaging.

dose reduction by growing number of examinations.

According to European Community and American guidelines, radiation dose reduction becomes a constant public concern because of the fast growing use of CT, leading to the principle of keeping dose exposure as low as reasonably achievable ${ }^{(31)}$. The experimental study on cadaver heads using a surface registration method provided our team with routine and convincing data to adapt reduced CAS-CT dose protocols in clinical practice.

Accuracy of CAS navigation depends on multiple interlinked factors: e.g. technical accuracy of the navigation system, CT slice thickness, image reconstruction algorithms, scanner technology, radiation dose and background noise in surface reconstruction ${ }^{(15,32)}$. Moreover, radiation dose exposure does not only vary with different CT scanners but also with the specific patient size and study purposes rendering direct transfer of CT protocols between different institutions problematic. Intra-operative tissue shift ${ }^{(33)}$ represents additional factors in the navigation error bill. Despite those influences, TRE dependent on surface registration was not limited by dose reduction used in current technology. It seems that the big difference in radiation dose absorption between soft tissue and air, as represented on the Hounsfield scale (Figure 4), are big enough to be resistant to diminishing signal-to-noise ratio and blurring of the CT image by dose reduction.

In contrast to soft tissue based navigation in neurosurgery ${ }^{(33)}$, FESS is based on bone windows with high intrinsic contrast, slice thickness of $1 \mathrm{~mm}$ and accuracy of instrumentation at about $1 \mathrm{~mm}$, resulting in a generally accepted range of accuracy around $2 \mathrm{~mm}$. Thus, FESS is especially suited for CT dose reduction because soft tissue shift during surgery plays less of a role than in neurosurgery and because the limit for resection is mostly represented by stable bone margins. The conspicuous skeletal margins are also more resistant to background noise than the delineation of fluid or soft tissue components in soft tissue surgery due to the higher gradient in tissue dose absorption reflected in the high gradients between bone and soft tissue on the Hounsfield scale (Figure 4).

In our study, the limits of dose reduction are not reached by diminishing technical registration accuracy but by reduction of image quality as perceived by the individual surgeon. Different subjective demands on picture quality for delineation of soft tissue components caused first problems at dose reduction from $9.6 \mathrm{mGy}$ to $6 \mathrm{mGy}$ CTDI. However, clinicians and radiologists had the tendency to oversee the fact that most navigation in FESS is based on bone window data sets and the actual high quality and oversized image on the screen in the radiology department did not correspond to the much smaller view with reduced angle and adverse light effect which are a fact in day-today OR routine.

A useful strategy to expose the patient to a reduced CAS-CT dose protocol was stratification of indications in diagnostic CT protocols for diagnosis of soft tissue process (e.g. abscess, tumor invasion) and in CAS-CT protocols for chronic hyperplastic sinusitis, especially in revision surgery and when pre-existent scans had to be repeated because they were not usable for the navigation system. The amount of radiation dose reduction is limited by the demands on picture quality and thrust ${ }^{(34)}$ by each surgeon and scanner type, which makes a direct transfer of our values to other institutions problematic ${ }^{(35)}$. However, it is promising to optimize dose reduction for any new scanner installed, for dose protocols which have not been revised over several years and if CAS-CTs are routinely performed at the same radiation dose as for diagnostic CT with contrast media.

Reviewing the last 12 months of our pragmatic approach of dose reduction, we reduced radiation doses for conventional standard situations in FESS for the majority of our patients (4 out of 5). The realized dose reduction from 65 to $9.6 \mathrm{mGy}$ CTDI corresponds to an estimated reduction from $2.2 \mathrm{mSv}$ to $0.3 \mathrm{mSv}$. At our institution, this saving of $1.9 \mathrm{mSv}$ is comparable to 19 potential future thorax x-rays or almost a cranial CT (i.e. $0.1 \mathrm{mSv}$ or $2 \mathrm{mSv}$, respectively).

\section{CONCLUSIONS}

Current CT technology provides the surgeon with detailed and excellent picture quality, however, at the cost of radiation dose exposure. To reduce this iatrogenic hazard, bone window based FESS offers a good opportunity for dose reduction if the need for high tissue contrast or contrast media application can be excluded. The limit of accuracy of today's clinically used surface registration technology is not reached using the dose reduced CAS-CT applied in our anatomical experiments. On the other hand, dose reduction leads to noisier pictures, which are subjectively perceived by each surgeon differently. Checking CT protocols for the precise indication according to suspected patholo- 
gy, individual patient size, date of last revision of the scanning protocols, newer scanner types or post-processing strategies have a great chance to reduce CAS-CT radiation dose for a significant portion of patients.

\section{ACKNOWLEDGEMENT}

The authors would like to thank Prof. Dr. med Dr. sc tech M. Kompis for his professional advisory help with the data interpretation and statistical illustrations and Eva Clamann for her corrections as a native speaker of American English. Moreover, we thank the Staff of the Institute of Diagnostic and Interventional Neuroradiology University of Bern, Inselspital, 3010 Bern, Switzerland for their various supports.

Marco Caversaccio is supported by the Swiss National Research Foundation for the project Computer-Aided and Image-guided Medical Interventions (www.co-me.ch) [51 NF 40-111383]

\section{REFERENCES}

1. Frush DP. Pediatric CT: Practical Approach to Diminish the Radiation Dose. Pediatr Radiol 2002; 43: 714-717.

2. Sternberg S. Unnecessary CT scans exposing patients to excessive radiation. USA Today. November 28, 2007.

3. Tesoriero HW. Worries mount over excessive CAT scans. The Wall Street Journal. November 2, 2006.

4. Brenner DJ, Hall EJ. Computed Tomography - Increasing Source of Radiation Exposure. N Engl J Med 2007; 357: 2277-2284.

5. Brenner DJ, Elliston DC, Hall EJ, et al. Estimated risks of radiation induced fatal cancer from pediatric CT. Am J Roentgenol 2001; 176: 289-296.

6. Caversaccio M, Eichenberger A, Häusler R. Virtual Simulator as a Training Tool for Endonasal Surgery. Am J Rhinol 2003; 17: 283290.

7. Caversaccio M, Langlotz F, Nolte LP, et al. Impact of a self-developed planning and self-constructed navigation system on skull base surgery: 10 years experience. Acta Otolaryngol 2007; 127: 403-407.

8. Hsu L, Fried MP, Jolesz FA. MR-guided endoscopic sinus surgery. Am J Neuroradiology 1998; 19: 1235-1240.

9. Tack D, Widelec J, De Maertelaer V, et al. Comparison between low-dose and standard-dose multidetector CT in Patients with suspected chronic sinusitis. Am J Radiol 2003; 181: 939-944.

10. Koller CJ, Eatough JP, Bettridge A. Variations in radiation dose between the same model of multislice CT scanner at different hospitals. Br J Radiol 2003; 76: 798-802.

11. Sohaib SA, Peppercorn PD, Horrocks JA, et al. The effect of decreasing $\mathrm{mAs}$ on image quality and patient dose in sinus $\mathrm{CT}$. Br J Radiol 2001; 74: 157-161.

12. Kearney SE, Jones $\mathrm{P}$, Meakin K, et al. CT scanning of the paranasal sinuses - the effect of reducing mAs. Br J Radiol 1997; 70: 1071-1074.

13. Nauer C, Eichenberger A, Dubach P, et al. CT Radiation Dose for Computer-Assisted Endoscopic Sinus Surgery: Dose Survey and Determination of Dose-Reduction Limits. Am J Neuroradiol 2009; 30: 617-622.

14. Knott PD, Batra PS, Citardi MJ. Computer aided surgery: concepts and applications in rhinology. Otolaryngol Clin N Am 2006; 39: 503522.

15. Labadie RF, Davis BM, Fitzpatrick JM. Image-guided surgery: what is the accuracy? Curr Opin Otolaryngol Head Neck Surg 2005; 13: 27-31.

16. Metson R, Cosenza M, Gliklich RE. The role of image-guided systems for head and neck surgery. Arch Otolaryngol Head Neck Surg 1999; 125: 1100-1104.

17. Hustedt H, Heermann R, Becker H. Contribution of low-dose CTscan protocols to the total positioning error in computer-assisted surgery. Comput Aided Surg 1999; 4: 275-280.
18. Stelter K, Andratschke M, Leunig A, et al. Computer-assisted surgery of the paranasal sinuses: technical and clinical experience with 368 patients, using the Vector Vision Compact system. J Laryngol Otol 2006; 120: 1026-1032.

19. Heermann R, Schwab B, Issing PR, et al. Navigation with the StealthStationTM in skull base surgery: an otolaryngological perspective. Skull Base 2001; 11: 277-285.

20. Caversaccio M, Giraldez JG, Thoranaghatte R, et al. Augmented reality endoscopic system (ARES): Preliminary Results. Rhinology 2008; 46: 156-158.

21. Balachandran R, Fitzpatrick M, Labdie RF. Accuracy of image-guided surgical systems at the lateral skull base as clinically assessed using bone-anchored hearing aid posts as surgical targets. Otol Neurotol 2008; 29: 1050-1055.

22. Hemmedinger SA, Jacobs JB, Lebowitz RA. Accuracy and Cost Analysis of Image-Guided Sinus Surgery. Otolaryngol Clin N Am 2005; 38: 453-460.

23. Schlaier J, Warnat J, Brawanski A. Registration Accuracy and Practicability of Laser-Directed Surface Matching. Computer Aided Surg 2002; 7: 284-290.

24. Grevers G, Leunig A, Klemens A, et al. Computerassistierte Chirurgie der Nasennebenhöhlen - Technologie und klinische Erfahrung mit dem VectorVision compact System an 102 Patienten. Laryngorhinotologie 2002; 56: 51-59.

25. Schmerber S, Chassat F. Accuracy evaluations of a CAS system: laboratory protocol and results with 6D localizers, and clinical experiences in otorhinolaryngology. Computed Aided Surg 2001; 6: 1-13.

26. Olson G, Citardi M. Image-guided functional endoscopic sinus surgery. Otolaryngology Head Neck Surg 2000; 123: 188-194.

27. Fried MP, Kleefield J, Gopal H, et al. Image-Guided endoscopic surgery: result of accuracy and performance in a multicenter clinical study using an electromagnetic tracking system. The Laryngoscope 1997; 107: 594-601.

28. Ramrath L, Vogt S, Jensen W et al. Computer- and robot-assisted stereotaxy for high-precision small animal brain. Biomed Tech (Berl) 2009; 54: 8-13.

29. Guggisberg AG, Dubach P, Hess CW. Motor evoked potentials from masseter muscle induced by transcranial magnetic stimulation of the pyramidel tract - the importance of coil orientation. Clin Neurophysiol 2001; 112: 2312-2319.

30. Jackman AH, Palmer JN, Chiu AG et al. Use of intraoperative CT scanning in endoscopic sinus surgery: A preliminary report. Am J Rhinol 2008; 22: 170-174.

31. Fox AJ. Use of the lowest necessary radiation dose. Am J Neuroradiol 2004; 25: 519.

32. Ecke U, Khan M, Maurer J, et al. Intraoperative Navigation in der Chirurgie der Nasennebenhöhlen und der vorderen SchädelbasisFehlerquellen und Störfaktoren. HNO 2002; 50: 928-934.

33. Raabe A, Krishnan R, Wolff R et al. Laser surface scanning for patient registration in intracranial image-guided surgery. Neurosurgery 2002; 50: 797-803.

34. Strauss G, Koulechov K, Röttger S, et al. Evaluation of a Navigation System for ENT with Surgical Efficency Criteria. Laryngoscope 2006; 116: 564-572.

35. Marmolya G, Wiesen EJ, Rauf Y, et al. Paranasal sinuses: Low-dose CT. Radiology 1991; 181: 689-691

P. Dubach

Department of Otorhinolaryngology

Head and Neck Surgery Inselspital

Bern University Hospital

and University of Bern

Bern, Switzerland

Tel: +41-31-372 1131

E-mail: patrick.dubach@insel.ch 